\title{
TRANSFUSION OF LABILE BLOOD PRODUCTS INTO NEONATOLOGY
}

\author{
F. BENNAOUI ${ }^{1,2}$, N. EL IDRISSI SLITINE ${ }^{1,2}$, F.M.R MAOULAININE ${ }^{1,2}$ \\ 1-Neonatal Intensive Care Department, Mohammed VI University Hospital and Research \\ 2-Team for Childhood, Health and Development, Marrakech School of Medicine, Cadi Ayyad University, \\ Marrakesh, Morocco.
}

\section{INTRODUCTION}

-Blood transfusion is a replacement therapy that involves transfusing the blood or one of its cellular or plasma components from one or more subjects called "donors" to one or more diseased subjects called "recipients".

The objective of this work is:

To evaluate the consumption of blood products in our service and to support the different clinical and biological situations leading to the practice of blood transfusion

\section{MATERIALS AND METHODS}

-This is a retrospective study of 60 observations of neonates who received one or more transfusions of one or more labile blood products collected in the Neonatal intensive care Unit. Mohammed VI, Marrakesh.

- Period: one year from January 1st to December 31 st, 2016

\section{RÉSULTATS}

-The age of hospitalized newborns ranged from one day to 44 days. (Figure 1)

-Our population consisted of 33 boys and 27 girls, a sex ratio of 1.22 .

-Transfected newborns were hospitalized in $38.3 \%$ for neonatal jaundice, while in $16.7 \%$ for anemic syndrome

- Blood transfusion was indicated in all transfused patients (100\%) (Figure 2.3).

- The main indications for transfusion of platelet pellet in our patients were thrombocytopenia in $62.5 \%$ of cases, followed by pancytopenia in $25 \%$ of cases and bleeding syndrome in $12.5 \%$ of cases

\section{DISCUSSION}

- Blood transfusion is common in neonatal intensive care unit. In fact, 50 to $90 \%$ of children of gestational age less than 32 weeks of amenorrhea or birth weight of less than $1500 \mathrm{~g}$ are transfused.

The rational use of blood and its components was only possible when the techniques of observation were sufficiently powerful to allow scientists to analyze blood accurately and accurately. Thus it was possible to explain calmly the role of each element of the blood but also its immunogenicity.

Figure 1: Distribution of cases by age

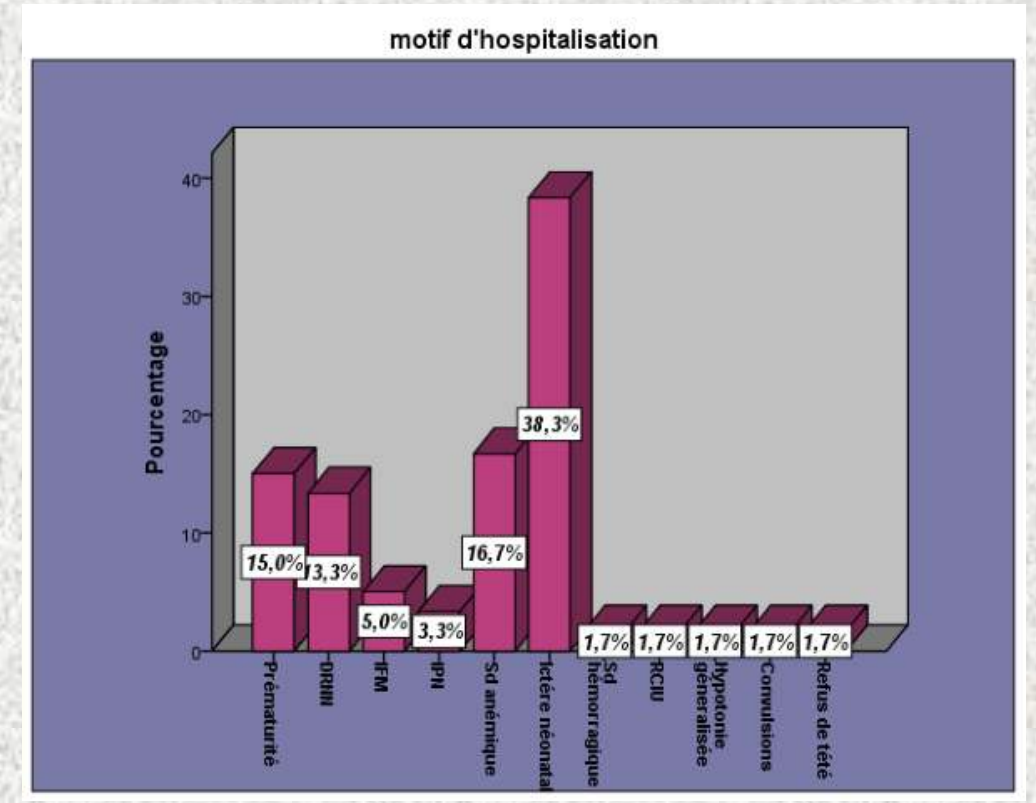

Blood transfusion is the implementation of all this knowledge.

Transfusion is known by knowing the specificities of the blood groups of the recipient and the donor, to avoid immunological accidents, but it is also transfused selectively, namely that the recipient, who does not necessarily need all the components of the blood complex tissue will only be provided with what it needs.

\section{CONCLUSION}

Transfusion practices have changed in recent years, the neonatal period remains a period when transfusion of labile blood products (especially red blood cell concentrates) is common, especially in low birthweight premature infants.

It is therefore important to be familiar Figure 3: Indication of transfusion of red blood cells. with the specific pathophysiological features of this age of life in order to reduce the risks of transfusion and to allow optimal efficacy of this therapy. 